\title{
The enigma in the heart of Ursa Minor
}

\author{
S. Demers ${ }^{1}$ and P. Battinelli ${ }^{2}$ \\ 1 Département de Physique, Université de Montréal, CP 6128, Succursale Centre-Ville, Montréal, \\ Québec H3C 3J7, Canada \\ 2 Osservatorio Astronomico di Roma Viale del Parco Mellini 84, 00136 Roma, Italy \\ e-mail: battinel@oarhp1.rm.astro.it
}

Received 18 June 2001 / Accepted 9 August 2001

\begin{abstract}
To investigate the physical nature of the observed deficiency of stars in the very center of the Ursa Minor Dwarf Spheroidal galaxy, we recently obtained JCMT-SCUBA $850 \mu \mathrm{m}$ photometry of this region. The observed void could be explained by $\approx 0.5 \mathrm{mag}$ of extinction. While the amount of dust needed to produce such absorption (under certain assumptions for the dust temperature and the $\beta$ exponent in the dust emissivity term) would yield a $5 \mathrm{mJy}$ signal - easily detectable by SCUBA - we found no signal at $850 \mu \mathrm{m}$, after $256 \mathrm{~min}$ of integration. This finding rules out the presence of a few solar masses of cold dust cloud as the explanation for the central lack of stars observed in Ursa Minor. We discuss possible explanations.
\end{abstract}

Key words. galaxies: individual: Ursa Minor - galaxies: ISM - ISM: dust, extinction

\section{Introduction}

Not so long ago, dwarf spheroidal (dSph) galaxies were believed to be simple systems made up of just one generation of old stars. Although some peculiarities were observed in their photographic CMDs, the stellar populations of the Local Group dSph galaxies were thought, in general, to be similar to those found in the globular clusters of the Milky Way. Surprises started to appear in the late seventies with the discovery of numerous carbon stars in the Fornax dSph which implied the presence of an intermediate-age stellar population (Demers \& Kunkel 1979). The low-mass Carina dSph was found to be of intermediate age (Mould \& Aaronson 1983) with multiple main-sequence turnoffs (Mighell 1990). HI gas was found in Phoenix (Carignan et al. 1991), making its classification ambiguous, and more recently, HI gas was also detected in Sculptor (Carignan et al. 1998) which is the prototype dwarf spheroidal galaxy. The latest surprise is a strange population, observed in the center of Sculptor by HurleyKeller et al. (1999), consisting in a spur of stars that extends from the main sequence turnoff. The evolution of dSph galaxies is clearly much more complex than previously believed.

In this context, three very recent papers, all based on HST observations, demonstrate the complexity of the evolution of the Ursa Minor dSph galaxy:

- Battinelli \& Demers (1999 hereafter B\&D) have confirmed the existence of a central density cusp

Send offprint requests to: S. Demers, e-mail: demers@astro.umontreal.ca
(Demers et al. 1995) and discovered a statistically significant deficiency of stars in the inner $13^{\prime \prime}$ (radius of $4 \mathrm{pc}$ ) of the center of UMi. Only 73 stars down to $V=26$ were counted in a region where a few hundred were expected based on an extrapolation of the surface density profile to the center of the galaxy. They proposed extinction by dust as a likely explanation for this lack of stars in the center;

- Feltzing et al. (1999; hereafter FGW) have found (in an off-center region) a number of blue stars that seems to define a blueward main sequence. The number of these blue stars is compatible with the frequency of blue stragglers derived by Olszewski \& Aaronson (1985). Since no such stars are observed in the FGW offset field, these blue stars are likely UMi members;

- Mighell \& Burke (1999; hereafter M\&B) present a $V$, $I$ CMD which shows that most of the UMi stellar population is similar to the ancient metal-poor Galactic globular cluster M 92. However, their CMD also shows a larger number of blue stars than seen by FGW - in a region that partially overlaps the $\mathrm{B} \& \mathrm{D}$ field. $\mathrm{M} \& \mathrm{~B}$ do not comment on the nature of these blue stars. This result may be indicative of the presence of a population similar to the spur stars seen in Sculptor by HurleyKeller et al. (1999). The placement of the M\&B UMi field had little overlap with the UMi void discovered by B\&D: only $34 \mathrm{M} \& \mathrm{~B}$ stars $(20<V<25)$ are found to lie within the void. Considerably more stars would be required to determine if there is a systematic color difference between the stars in the void and those outside of it. 
Moreover, Martinez-Delgado \& Aparicio (1999; hereafter MD\&A), using ground-based wide-field observations, have confirmed the presence of a strikingly well-populated blue plume interpreted by these authors as a tracer of an intermediate-age stellar population in UMi. Again, a comparison with a field well outside the UMi tidal radius shows that these blue stars are likely members of the UMi galaxy. Contrary to the conclusions of FGW, MD\&A estimate that this blue population is about three times more abundant than the expected number of blue stragglers.

Since evolved stars lose mass during their advanced stages of evolution, dust is expected - and has long been searched for - in the center of old stellar systems like globular clusters. However, attempts to find dust have too often been futile. At present, dust has been detected in only two clusters (47 Tuc: Origlia et al. 1996; NGC 6356: Hopwood et al. 1998) which is well below the expected number. As mentioned above, the stellar content of UMi is quite similar to one of the typical Galactic metal-poor globular clusters which suggests that dust could also be present in the UMi dSph.

The lack of dust in globular clusters is most probably due to their frequent passage through the Galactic disk. On the other hand, several factors suggest that it might be reasonable to expect that any dust in $\mathrm{UMi}$, if it exists at all, would have a much greater chance of not be removed or destroyed because: i) UMi may never pass through the Galactic disk; ii) UMi's lower central stellar density means less efficient gas/dust removal by radiative pressure; iii) UMi is more massive than a typical cluster thus its gravitational well can retain more dust; and iv) UMi is quite distant compared to Galactic clusters which implies that both the hot halo gas and galactic radiation will be far less efficient mechanisms for dust grain destruction.

\section{Observations and photometry}

The data were obtained, on 2000 February 1st, using SCUBA in photometry mode on the 15-m James Clerk Maxwell Telescope (JCMT). The very center of the Ursa Minor galaxy, located at $\mathrm{RA}=15^{\mathrm{h}} 08^{\mathrm{m}} 41^{\mathrm{s}} .1$ and decl. $=$ $67^{\circ} 12^{\prime} 35^{\prime \prime}$ (J2000) was observed simultaneously with the $450 \mu \mathrm{m}$ and $850 \mu \mathrm{m}$ arrays. The SCUBA primary calibrator, Uranus, located $70^{\circ}$ from UMi was used, along with secondary sources selected from the JCMT catalogue to calibrate fluxes and check pointing and focusing. The beam-widths corresponding to the 850 and $450 \mu \mathrm{m}$ bolometers are $14^{\prime \prime}$ and $8^{\prime \prime}$ respectively which projected on Ursa Minor are equal and smaller than the diameter of the void. The observations were done by integrating both on and off the source. A East-West chop throw of $60 \operatorname{arcsec}$ at $8 \mathrm{~Hz}$ was adopted. The observations consisted in a series of $18 \mathrm{~s}$ integrations with skydips and pointing taken at approximately every hour. The total on-source time was $256 \mathrm{~min}$. The data were reduced on line using, SURF (Jenness \& Lightfoot 1998). In the SCUBA photometer mode only the central bolometer of each array is used to observe the target. The outer bolometers are thus used to better correct the sky variations not seen by the chopping. In the $14^{\prime \prime}$ wide beam of SCUBA we measured $0.285 \pm 0.856 \mathrm{mJy}(S / N=0.33)$ for the $850 \mu \mathrm{m}$ beam and a negative flux with even a larger error for the $450 \mu \mathrm{m}$ beam.

\section{Discussion}

\subsection{Upper limit to the amount of dust in the core of Ursa Minor}

There is no source, in the IRAS faint sources catalog (Moshir et al. 1992), in the direction of the core of Ursa Minor. This fact implies that the dust, if any, must be too cold to emit at wavelengths shorter than $100 \mu \mathrm{m}$, or that there is not more than $0.5 M_{\odot}$ of dust at a temperature higher than $25 \mathrm{~K}$. B\&D have estimated the central stellar density of Ursa Minor to be $\approx 3 \mathrm{stars} \mathrm{pc}^{-3}$, thus the stellar radiation field is low and the dust, if in equilibrium with the radiation field, would be a quite low temperatures. Models of dust heating in various environments (Mezger et al. 1982) show that the lacks of OB stars rules out temperatures greater than $25 \mathrm{~K}$. Interstellar dust can however be heated in the vicinity of red giants, as Le Bertre et al. (1998) observations have shown. There is no red giant in the central region of Ursa Minor. The brightest star in the void is an horizontal branch star.

Adopting Zinnecker's et al. (1992) formulation (Eq. (5)) we can estimate formally the mass of the dust cloud producing the observed flux, eventhough the uncertainty attached to the above figure suggests that the flux may very well be nearly null. Only two parameters need to be selected: the temperature of the dust and the emissivity coefficient $\beta$. It is believed that if dust exists in the center of dwarf spheroidal galaxies it should be of different nature than the dust in the solar neighbourhood. Such dust should be similar to circumstellar dust rather than interstellar dust. This type of dust is believed to be amorphous carbon, therefore a $\beta=1.0$ seems appropriate (Knapp et al. 1993; Mennella et al. 1995). For a temperature of $25 \mathrm{~K}$, the above flux yields a dust + gas mass of $4.5 M_{\odot} \pm 15.3$ and a larger mass of $21 M_{\odot} \pm 70$ for a lower temperature of $10 \mathrm{~K}$. This is computed taking the distance of UMi to be $68 \mathrm{kpc}(\mathrm{M} \& \mathrm{~B})$. This mass would be within the JCMT beam of $14^{\prime \prime}$ or $4.4 \mathrm{pc}$. The larger mass, corresponding to the low temperature, would produce a visual extinction of $A_{V} \approx 0.1$. These values are compatible with zero dust and gas in the core of UMi.

\subsection{The gas content of Ursa Minor}

The disappointing absence of dust in the very center of UMi is however in good agreement with the recent Young's (2000) NRAO observations. In the central part of UMi Young found no more than $310 M_{\odot}$ of neutral hydrogen. The $F W H M$ of the $140 \mathrm{ft}$ NRAO telescope is $21^{\prime}$, much larger than our 14" SCUBA beam. If we assume, as 
reasonable, that $\mathrm{HI}$ is spatially distributed similarly to what the UMi stellar component is, we can adopt the radial stellar density profile given by $B \& D$ to evaluate the amount of HI expected in our SCUBA beam. In this way, we estimate that $\approx 0.5 M_{\odot}$ of $\mathrm{HI}$ is confined in our $14^{\prime \prime}$ disc, this (for any reasonable gas-to-dust mass ratio) implies no detectable dust in the very center of UMi. Finally, we stressed that the above amount of HI gas in the very center of UMi must be considered an optimistic upper value since: i) Young's detection of $310 M_{\odot}$ is an upper limit; ii) the adopted King profile $(B \& D)$ is calibrated on star counts in the inner part of UMi and thus results much steeper than other determinations, like e.g. in Irwin \& Hatzidimitriou (1995).

\subsection{Expected dust production during the life time of UMi}

The central stellar density of Ursa Minor, as evaluated by $\mathrm{B} \& \mathrm{D}$, is $\sim 10^{5}$ less than quoted values based on models of globular clusters (Illingworth \& King 1977). Taking into account the undetected white dwarfs in UMi, we can safely say that its central density is thousands of time less than the one of a typical globular cluster. Therefore, the amount of dust resulting from stellar mass loss during latest evolutionary phases will be correspondingly much less than what is expected in globular clusters. Indeed, Carrera et al. (2001) have calculated that the total mass converted into stars during the entire lifetime of UMi and currently lying within its inner $18^{\prime}$ radius is $1.8 \times 10^{5} M_{\odot}$. If, as above, we assume that this mass is radially distributed following the stellar density profile obtained by B\&D, we can estimate that there are barely $500 M_{\odot}$ of stars in the inner $14^{\prime \prime}$ region, corresponding to the JCMT beam. This mass compares favorably with the number of stars in this central region. From the B\&D stellar density quoted above we calculate that there are 879 stars in this small volume.

If we assume that the fraction of the mass returned into the interstellar space is 0.2 , then there should be $\sim 100 M_{\odot}$ of gas and dust in the center of UMi. For a gas to dust ratio of 100 this would barely amount to $1 M_{\odot}$ of dust.

\subsection{The orbit of Ursa Minor}

From her proper motion study, Schweitzer (1996) estimated that the perigalacticon distance of Ursa Minor is $\sim 43 \mathrm{kpc}$ and its apogalacticon distance is $\sim 81 \mathrm{kpc}$. Similar distances, namely 49 and $110 \mathrm{kpc}$, were calculated by Lynden-Bell \& Lynden-Bell (1995). We can thus say in confidence that Ursa Minor has never come close to the galactic disc. Therefore one cannot invoke the interaction with the galactic disc to explain the lack of dust.

We thus conclude that the non detection of a $850 \mu \mathrm{m}$ signal is due to the low stellar density which yielded so little dust and not due to its removal by external mechanism. The deficiency of stars at the very centre of UMi is still unexplained.
Acknowledgements. The authors are particularly grateful to Pierre Bastien who provided his programme to compute the dust mass from the SCUBA flux. We would like to thank Henry Matthews for his advises on the JCMT operation. The James Clerk Maxwell Telescope is operated on behalf of the Particle Physics and Astronomy Research Council of the United Kingdom, the Netherlands Organisation for Scientific Research and the National Research Council of Canada. This research is partially funded (S. D.) by the Natural Science and Engineering Council of Canada.

\section{References}

Battinelli, P., \& Demers, S. 1999, AJ, 117, 1764 (B\&D)

Carignan, C., Demers, S., \& Coté, S. 1991, ApJ, 381, L13

Carignan, C., Beaulieu, S., Coté, S., Demers, S., \& Mateo, M. 1998, AJ, 116, 1690

Carrera, R., Aparicio, A., \& Martinez-Delgado, D. 2001, private communication

Demers, S., \& Kunkel, W. E. 1979, PASP, 91, 761

Demers, S., Battinelli, P., Irwin, M. J., \& Kunkel, W. E. 1995, MNRAS, 274, 491

Feltzing, S., Gilmore, G., \& Wyse, R. F. G. 1999, ApJ, 516, L17 (FGW)

Hopwood, M. E. L., Evans, A., Penny, A., \& Eyres, S. P. S. 1998, MNRAS, 301, 30

Hurley-Keller, D., Mateo, M., \& Grebel, E. K. 1999, ApJ, 503, L25

Illingworth, G., \& King, I. R. 1977, ApJ, 218, L109

Irwin, M. J., \& Hatzidimitriou, D. 1995, MNRAS, 277, 1354

Jenness, T., Lightfoot, J. F. 1998, Starlink User Notes 216

Knapp, G. R., Sandell, G., \& Robson, E. I. 1993, ApJS, 88, 173

Le Bertre, T., Lagache, G., Mauron, N., et al. 1998, A\&A, 335, 287

Lynden-Bell, D., \& Lynden-Bell, R. M. 1995, MNRAS, 275, 429

Martinez-Delgado, D., \& Aparicio, A. 1999, in The Stellar Content of Local Group Galaxies, ed. P. Whitelock, \& R. Cannon, IAU Symp., 192, 179 (MD\&A)

Mennella, V., Colangeli, L., \& Bussoletti, E. 1995, A\&A, 295, 165

Mezger, P. G., Mathis, J. S., \& Panagia, N. 1982, A\&A, 105, 372

Mighell, K. J. 1990, A\&AS, 82, 1

Mighell, K. J., \& Burke, C. 1999, AJ, 118, 366 (M\&B)

Moshir, M., et al. 1992, Explanatory Supplement to the IRAS Faint Source Survey, Vers. 2, JPL D-10015 (Pasadena: JPL) (FSC)

Mould, J.R., \& Aaronson, M. 1983, ApJ, 273, 530

Olszewski, E. W., \& Aaronson, M. 1985, AJ, 90, 2221

Origlia, L., Ferraro, F. R., \& Fusi Pecci, F. 1996, MNRAS, 280, 572

Schweitzer, A. E. 1996, Ph.D. Thesis, University of WisconsinMadison

Young, L. M. 2000, MNRAS, 280, 572

Zinnecker, H., Bastien, P., Arcoragi, J.-P., \& Yorke, H. W. 1992, A\&A, 265, 726 